\title{
Advances in electroanalysis, sensing and monitoring in molten salts
}

\author{
Damion K. Corrigan, ${ }^{a}$ Justin P. Elliott, ${ }^{a}$ Ewen O. Blair, ${ }^{b}$ Simon J. Reeves, ${ }^{a}$ \\ Ilka Schmüser, ${ }^{b}$ Anthony J. Walton ${ }^{b}$ and Andrew R. Mount ${ }^{\star a}$
}

Received 6th January 2016, Accepted 11th February 2016

DOI: $10.1039 / c 6 f d 00002 a$

Microelectrodes have a number of advantages over macroelectrodes for quantitative electroanalysis and monitoring, including reduced $i R$ drop, a high signal-to-noise ratio and reduced sensitivity to convection. Their use in molten salts has been generally precluded by the combined materials challenges of stresses associated with thermal cycling and physical and corrosive chemical degradation at the relatively high temperatures involved. We have shown that microfabrication, employing high precision photolithographic patterning in combination with the controlled deposition of materials, can be used to successfully address these challenges. The resulting molten salt compatible microelectrodes (MSMs) enable prolonged quantitative microelectrode measurements in molten salts (MSs). This paper reports the fabrication of novel MSM disc electrodes, chosen because they have an established ambient analytical response. It includes a detailed set of electrochemical characterisation studies which demonstrate both their enhanced capability over macroelectrodes and over commercial glass pulled microelectrodes, and their ability to extract quantitative electroanalytical information from MS systems. MSM measurements are then used to demonstrate their potential for shedding new light on the fundamental properties of, and processes in, MSs, such as mass transport, charge transfer reaction rates and the selective plating/stripping and alloying reactions of liquid $\mathrm{Bi}$ and other metals; this will underpin the development of enhanced MS industrial processes, including pyrochemical spent nuclear fuel reprocessing.

\section{Introduction}

Molten salts (MSs) are a key constituent of a number of industrial processes, including metal refining, ${ }^{1}$ pyrochemical reprocessing of spent nuclear fuel, ${ }^{2-5}$ molten salt batteries ${ }^{6}$ and catalysis. ${ }^{7,8}$ Chloride melts are the MS of choice for a wide range of applications, due to their relatively low melting points and viscosities, their complexing ability, and their reduced corrosion compared to fluoride melts. A particularly intensively studied chloride system is the lithium

${ }^{a}$ EaStCHEM, School of Chemistry, The University of Edinburgh, King's Buildings, Edinburgh, EH9 3FJ, UK. E-mail:a.mount@ed.ac.uk

${ }^{b} S M C$, School of Engineering, The University of Edinburgh, King's Buildings, Edinburgh, EH9 3FF, UK 
chloride-potassium chloride eutectic (LKE) which has a relatively low melting point of $625 \mathrm{~K}$ at the eutectic composition (59 $\mathrm{mol} \% \mathrm{LiCl}: 41 \mathrm{~mol} \% \mathrm{KCl}$ ), a relatively high ionic conductivity of $1.57 \mathrm{~S} \mathrm{~cm}^{-1}$ at $723 \mathrm{~K}$ (ref. 5) and a wide electrochemical window of $3.62 \mathrm{~V}$, within which a wide range of reactions can be studied. $^{5}$

As a result of its industrial importance, particularly in the nuclear sector, there has been a desire to develop analysis techniques suitable for MS systems, which address the challenges of withstanding the high temperatures and corrosive nature of the melts when they contain reactive species. ${ }^{9-11}$ Electrochemical methods have typically been practically limited to macroelectrode measurements $^{12-20}$ and therefore suffer from a low signal-to-noise ratio, a time-dependent signal, $i R$ distortion of the response and sensitivity to convection. ${ }^{21}$ These effects at best complicate and often preclude quantitative analysis. Optical spectroscopies suffer from the disadvantages of the broad emissions of many of the target analytes, difficulties in identifying multiple analytes and corrosion of sampling windows. ${ }^{10}$

When considering electroanalysis, microelectrodes can be fabricated using a number of methods and have well-established advantages under ambient conditions of high signal-to-noise ratios, and steady-state (time-invariant) and quantitative signals which are relatively insensitive to convection when compared to macroelectrodes. ${ }^{22,23}$ They have therefore been developed for ambient and nearambient sensing applications ${ }^{24}$ and for use in the monitoring of industrial processes. ${ }^{25}$ However, there has been a significant technological barrier to the development of similar microelectrode sensors for reproducible and quantitative MS analysis and monitoring. For example, microelectrodes fabricated by drawing a wire encapsulated in glass and then exposing the end, have been employed with limited success, as this has been shown to result in initial and time-dependent variation of the response. ${ }^{26}$ The initial variability can be attributed to differences in the wire size and cross section due to variation in the wire drawing process, which as a result requires optical and/or electrochemical characterization of electrode size and shape. The time-dependent variation in the MS can be attributed to one or more of: the tendency for chemical attack of the glass, which compromises the electrode insulation; the differential thermal expansion of the microelectrode materials, which causes materials stress and cracking; and the chemical corrosion of the electrode, which can cause it to progressively recess with time. All of these effects cause a progressive change in effective electrode area and the diffusion regime, which precludes the effective deployment of microelectrodes in MS media for physicochemical analysis.

Previously, we have reported the development and characterisation of an MSM designed to overcome the above issues. ${ }^{21,27-29}$ In this study we have built on our previous work, employing our established photolithographic fabrication techniques to produce novel disc-shaped MSMs with precisely controlled dimensions and geometries. Disc microelectrodes have been chosen due to their well-established and quantitative ambient analytical response. ${ }^{29}$ We first establish whether the microelectrode response of the disc MSMs under ambient aqueous conditions is similar to other microelectrodes and then compare and contrast the performance of these devices in the LKE MS $^{\mathbf{2 1}}$ both with macroelectrodes and with the ambient microelectrode response. This paper has three main aims: (1) to clearly demonstrate the enhanced capabilities of the MSM in MS by benchmarking 
against a traditional glass pulled microelectrode, (2) to quantitate and explain the analytical response and extract physicochemical parameters in order to gain fundamental insight into the behaviour of the LKE molten salt system and (3) to investigate the potential for gaining fundamental insight into industrially relevant molten salt processes through MSM measurements. In this work we have concentrated on the characterisation of mass transport and charge transport in redox processes, along with the plating/stripping of metals and the formation of metal alloys. These are essential steps in the development of molten salt industrial processes, including pyrochemical spent nuclear fuel reprocessing, which we are developing through UK (the EPSRC REFINE programme) and EU (SACSESS programme) activities.

\section{Materials and methods}

\subsection{Design and fabrication of the electrode devices}

The disk MSM devices were produced using reliable, well-characterised siliconbased microfabrication processes (Fig. 1). First, a $500 \mathrm{~nm}$ thick layer of silicon dioxide insulation (Fig. 1A) was grown on the silicon substrate. Next, a $20 \mathrm{~nm}$ thick film of titanium nitride was deposited onto the surface as an adhesion layer for the electrode metal (which was either a $200 \mathrm{~nm}$ thick layer of tungsten or a 50 $\mathrm{nm}$ thick layer of platinum) (Fig. 1B). Patterning of the electrode metal layer was then achieved by reactive ion etching, using photolithographic masking to prevent the exposure of a band of metal around the perimeter of each device when diced (Fig. 1C). Subsequent deposition of a $500 \mathrm{~nm}$ thick layer of silicon nitride produced a top insulator (Fig. 1D), which was again patterned using photolithographic masking and reactive ion etching of the insulator to expose the bond pad (Fig. 1E) and the microelectrode (Fig. 1F). Fig. 1G shows a sketch of a completed device.

A

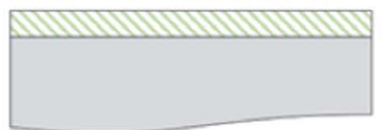

B

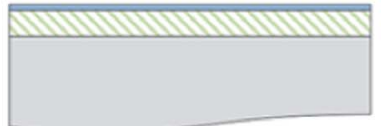

C

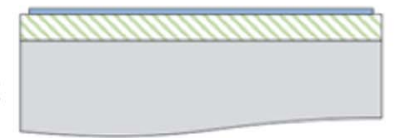

silicon substrate $\square$ silicon dioxide
D

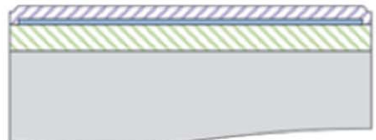

G

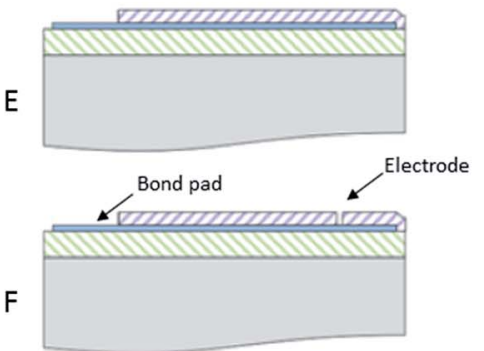

metallisation

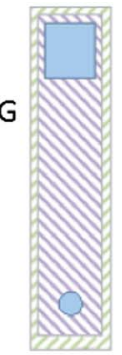

Fig. 1 Schematic showing cross-sections of the fabrication processes involved in producing the MSM (A-F) and a representative top view of a finished device with the microdisc (near the bottom) enlarged for clarity $(G)$. 


\subsection{High temperature measurements in LKE}

Experiments using LKE were conducted at fixed temperatures within the range $673-773 \mathrm{~K}$ in a glassy carbon crucible sealed inside a quartz cell within a vertical tube furnace (Carbolite, Derbyshire, UK). Tungsten wire (Goodfellow, Cambridge, $\mathrm{UK}$ ) was employed as the counter electrode with a $1 \% \mathrm{AgCl} / \mathrm{Ag}$ electrode used as the reference electrode. The latter was prepared by encapsulating a silver wire in LKE containing $1 \%$ by mass silver(I) chloride (Sigma Aldrich, Poole, Dorset, UK) in a mullite tube (Multilab, Newcastle upon Tyne, UK). All potentials, E, applied to the working electrodes and quoted in this paper are with respect to this reference electrode. For each experiment, anhydrous analytical grade salts of $\mathrm{LiCl}$ and $\mathrm{KCl}$ (Sigma Aldrich, Poole, Dorset, UK) were mixed at the eutectic composition (a molar ratio of $7: 5$ ) in a glassy carbon crucible and then melted in vacuo under dry Argon to form LKE. The microelectrode (connected to a tungsten wire via a crocodile clip with the connector sealed in ceramic) was deployed through a port in the cell lid as in previous work.

\subsection{Ambient aqueous benchmarking measurements}

For ambient aqueous control experiments, the disc MSM was employed as the working electrode, with a saturated calomel reference electrode (SCE) and platinum gauze counter electrode. All aqueous solutions were prepared with deionised water (Millipore MilliQ). Electrochemical experiments were typically performed using background electrolyte solutions of aqueous potassium chloride (Fisher Scientific, lab reagent grade, $>99 \%$ purity), with redox experiments using potassium ferricyanide, $\mathrm{K}_{3}\left[\mathrm{Fe}(\mathrm{CN})_{6}\right]$ (Fisons, SLR reagent grade, $>99 \%$ purity) in background electrolyte. All solutions were thoroughly degassed using pure shield argon (BOC) for at least 20 minutes prior to experimentation.

All experiments in 2.2 and 2.3 were performed using a PC-controlled Autolab PGSTAT12 potentiostat (EcoChemie, Utrecht, Netherlands) and results were analysed using GPES 4.9 and NOVA 1.11 software (EcoChemie), with iterative fitting carried out using Origin 2015 (OriginLab). EIS experiments were typically carried out by applying a $10 \mathrm{mV}$ rms amplitude sinusoid superimposed on the dc potential, $E_{\mathrm{DC}}$, using logarithmically spaced frequencies between $100 \mathrm{kHz}$ and 0.1 $\mathrm{Hz}$.

\section{Results and discussion}

\subsection{Demonstrating the enhanced fidelity of the disc MSM}

In previous work, assessments have been made for the robustness, possibilities for quantitation and measurement errors associated with ambient aqueous measurements on square MSMs. ${ }^{21}$ In terms of the measured steady-state currents, it was found that typical intra-experimental errors were relatively low, with the variation in the mass transport limiting current for one electrode in a solution of a given concentration across repeated measurements being less than $1.4 \%$, and that for different electrodes of the same nominal edge length being less than $4.2 \%$. In both cases these reported errors were likely to be overestimates, as the largest errors involved measurements using volatile redox couples, which results in uncertainty associated with their concentration. ${ }^{21}$ Errors from ambient studies involving glass pulled microelectrodes can be $\sim 10 \%$ (ref. 24 ) highlighting the 
benefit of the microfabrication approach and also the fidelity of the MSM devices in the melt. In this study, as disc MSMs have been fabricated and characterised for the first time, the response and lifetime of these MSMs in LKE is initially contrasted with a commercially available glass pulled tungsten "cone" microelectrode, which has a base diameter of the exposed cone of $26 \mu \mathrm{m}$ (see Fig. 2A).

The quantitative response of cone microelectrodes has been established as: ${ }^{30}$

$$
i_{\mathrm{L}}=4 n F D c r\left(1+q H^{p}\right)
$$

where $i_{\mathrm{L}}$ is the mass transport limiting current, $n$ is the number of electrons transferred, $D$ is the diffusion coefficient, $F$ is Faraday's constant, $c$ is the bulk concentration, $r$ is the cone base radius, $q$ is $0.30611, H$ is the aspect ratio of the cone height to base radius and $p$ is 1.114 .

However, over the course of ten minutes in LKE these devices were observed to progressively physically deteriorate, with the etching and removal of the insulating glass seen to be the most prominent failure mechanism (see Fig. 2B-D). Thus, the exposed electrode area clearly enlarged with time. Given this, it is not
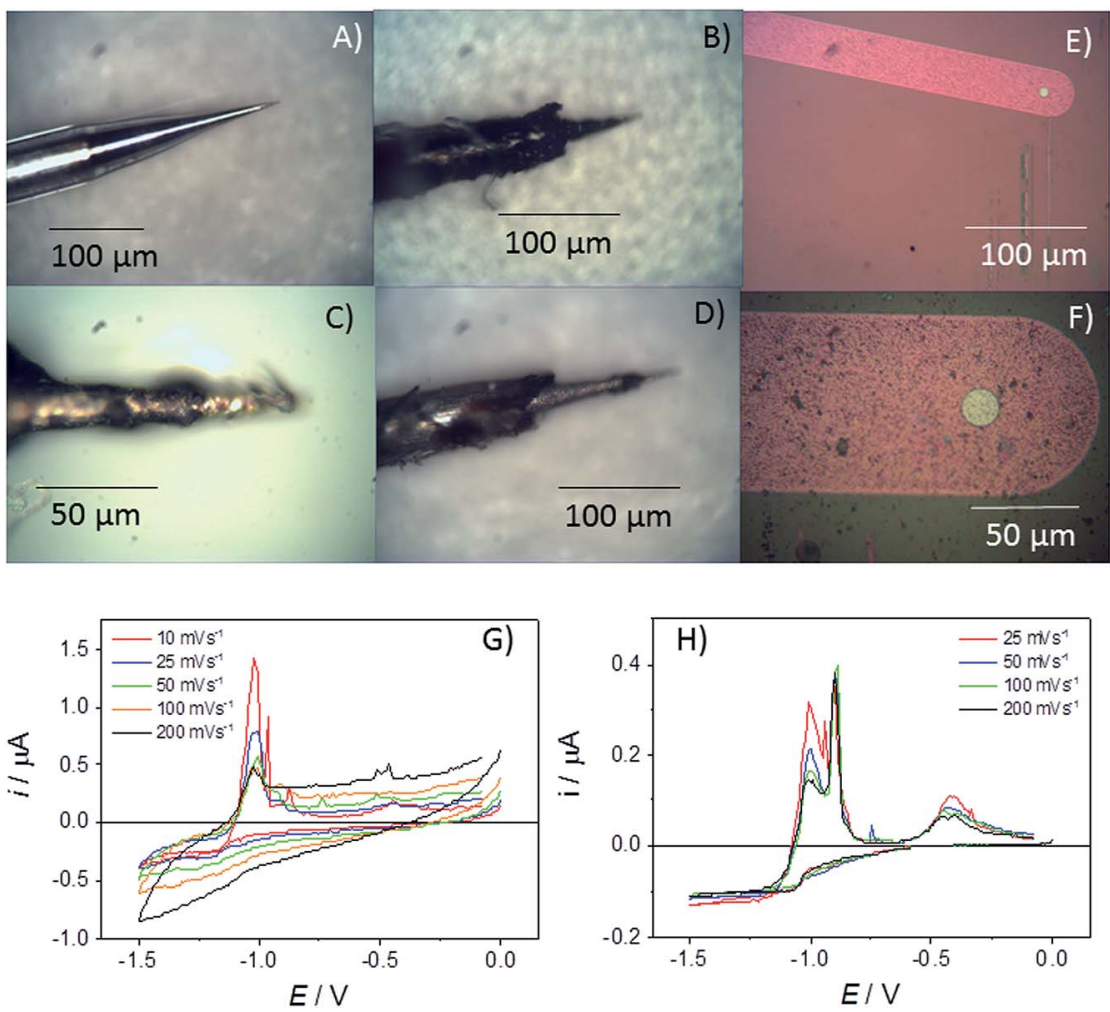

Fig. 2 Images of a commercial microelectrode (A) prior to immersion in LKE and (B-D) following progressive electrochemical cycling in $\mathrm{LKE}+5 \mathrm{mM} \mathrm{ZnCl}_{2}$. (E \& F) Images of a Pt $r$ $=10 \mu \mathrm{m}$ diameter disc MSM, (E) before measurement in LKE and (F) following $30 \mathrm{~min}$ of electrochemical cycling in LKE (see $\mathrm{H}$ ). (G) CVs recorded at the given range of sweep rates using the glass pulled tungsten microelectrode in LKE $+5 \mathrm{mM} \mathrm{ZnCl} 2$ at $773 \mathrm{~K}$. (H) CVs recorded using the $r=10 \mu \mathrm{m} \mathrm{Pt} \mathrm{MSM} \mathrm{in} \mathrm{LKE}+5 \mathrm{~mm} \mathrm{ZnCl}_{2}$ at $773 \mathrm{~K}$. 
surprising that the cyclic voltammograms (CVs) observed for $\mathrm{Zn}^{2+}$ reduction in LKE showed a change in response with scan rate and time (Fig. 2G). The observed increasing capacitance, and increasing and non-steady-state currents, together indicates the absence of characteristic microelectrode behaviour and an increasing exposed electrode area attributable to progressive insulator compromise. In contrast an $r=10 \mu \mathrm{m}$ Pt MSM employed for the same measurement showed no appreciable visible deterioration and produced a stable and characteristic microelectrode response, with the zinc reduction plating currents observed at $E<-0.5 \mathrm{~V}$ being invariant with time or sweep rate (Fig. $2 \mathrm{H}$ ). The resulting diffusion coefficient for $\mathrm{Zn}^{2+}$ in LKE of $2.85 \times 10^{-5} \mathrm{~cm}^{2} \mathrm{~s}^{-1}$, obtained from the mass transport limited current for this reduction, and the established analytical response of a disc microelectrode:

$$
i_{\mathrm{L}}=4 n F D c r
$$

is in good agreement with the literature and previous measurements with MSMs. ${ }^{21,31}$ In this case it was possible to obtain a stable electrochemical response for thirty minutes, over which the measured steady-state currents varied within the error limit previously established for square MSMs. After thirty minutes the experiment was terminated and there was no visible damage to the MSM (Fig. 2F). This therefore confirms the fidelity of the disc MSMs and their applicability to LKE MS measurement and analysis at least over these time scales.

\subsection{Quantitative physicochemical parameterisation using disc MSMs and soluble redox couples in LKE MS}

3.2.1 CV and chronoamperometric studies. Our previous work with solutionbased reactions ${ }^{21}$ focused on the initial demonstration of microelectrode performance in LKE solutions, with particular emphasis on comparison with macroelectrodes. The macroelectrode response was shown to suffer from convection and there were significant peak distortions in the CV, due to an $i R$ drop. In contrast, consistent with the disc MSM measurements in Fig. 2, convective effects were shown to be negligible in the MSM CV response; $i R$ effects were also shown to be absent by using modified Tafel analysis. ${ }^{21}$ This gives credit to the notion that MSMs should contain inherent analytical advantages over macroelectrodes in molten salts. ${ }^{21,27}$ In this study, the quantitative electrochemical behaviour of the $\mathrm{Eu}(\mathrm{III} / \mathrm{II})$ system is established in LKE using the disc MSM system. The Eu(III/II) system was chosen because it is a commonly employed model redox system representative of lanthanide species. ${ }^{10,12,32}$ Fig. 3A shows a typical CV recorded using an $r=25 \mu \mathrm{m}$ Pt disc MSM.

In chloride melts, $\mathrm{Eu}(\mathrm{III})$ has been shown to react according to the following scheme: ${ }^{32}$

$$
2 \mathrm{EuCl}_{3} \rightleftharpoons 2 \mathrm{EuCl}_{2}+\mathrm{Cl}_{2}
$$

It is clear that both oxidation and reduction waves can be seen on the CV, centred around $E=+0.4 \mathrm{~V}$, which arise from $\mathrm{Eu}(\mathrm{II})$ and $\mathrm{Eu}(\mathrm{III})$ species respectively, which we attribute to the effects of the above reactions in LKE (actually, the proportion of $\mathrm{Eu}(\mathrm{III})$ and $\mathrm{Eu}(\mathrm{II})$ is likely to vary between experiments with the 

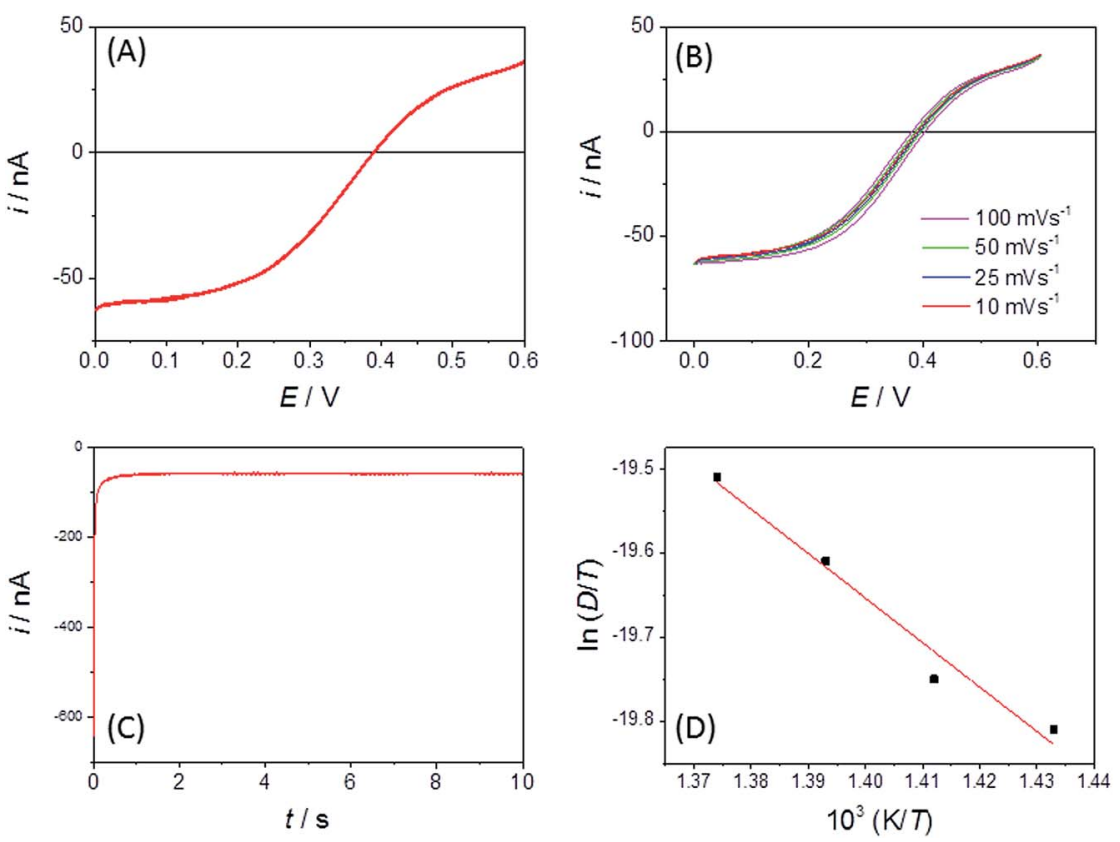

Fig. 3 (A) Typical CV obtained using a Pt disc $(r=25 \mu \mathrm{m}) \mathrm{MSM}$ in LKE $+19 \mathrm{mM} \mathrm{EuCl}_{3}$ (initial concentration) at a sweep rate of $10 \mathrm{mV} \mathrm{s}^{-1}$ and a temperature of $703 \mathrm{~K}$. (B) Corresponding $\mathrm{CVs}$ at the variety of given sweep rates. (C) Corresponding chronoamperometry (current, $i$ versus time, $t$ ) transient recorded using an $r=25 \mu \mathrm{m}$ Pt disc in LKE by stepping from $E=$ $+0.5 \mathrm{~V}$ to $0 \mathrm{~V}$ at $t=0$. (D) Arrhenius plot of $\ln (D / T)$ versus $1 / T$ used to obtain the activation energy for the diffusion of $\mathrm{Eu}^{3+}$ in LKE, where $D$ is the diffusion coefficient and $T$ is the temperature. The red line is the linear regression line of best fit.

concentration of dissolved chlorine in, and/or the overall oxidising environment of, the LKE). Reassuringly, the CV has a wave-like form characteristic of a microelectrode response, along with the characteristic microelectrode sweep rate independence (Fig. 3B) (there is only a small additional capacitive current whose importance increases as expected with sweep rate, leading to a slightly increased separation between forward and backward scans). Previous work ${ }^{32}$ has shown the diffusion coefficients for $\mathrm{Eu}(\mathrm{III})$ and $\mathrm{Eu}(\mathrm{II})$ to be comparable. Assuming therefore a global diffusion coefficient, $D$, and taking $i_{\mathrm{L}}$ as the difference in oxidation and reduction mass transport limited current and $c=19 \mathrm{mM}$, it is possible to calculate this global diffusion coefficient from eqn (2) as $(5.0 \pm 0.2) \times 10^{-6} \mathrm{~cm}^{2}$ $\mathrm{s}^{-1}$ in LKE at $703 \mathrm{~K}$; for any $\mathrm{CV}$, the combined heights of the oxidation and reduction waves (Fig. 2A and B) or the steady-state chronoamperometric currents observed at long times when carrying out both mass transport limited oxidation and reduction (e.g. Fig. 2C) through eqn (1) can then be used to determine the concentrations of $\mathrm{Eu}(\mathrm{III})$ and $\mathrm{Eu}(\mathrm{II})$. Fig. 3D shows an Arrhenius plot of $\ln (D / T) v s$. $1 / T$ derived from the global diffusion coefficient data obtained using the MSMs at various temperatures (note that this plot assumes the validity of the StokesEinstein equation ${ }^{33} D=\frac{k T}{6 \pi \eta r_{\mathrm{h}}}$, where $k$ is the Boltzmann constant, $r_{\mathrm{h}}$ is the effective radius of the diffusing species and $\eta$ is the viscosity of the MS, which is 
assumed to vary with temperature according to $\eta=\eta_{0} \exp \left[\frac{E_{\mathrm{a}}}{k T}\right]$, where $\eta_{0}$ is a constant and $E_{\mathrm{a}}$ is the activation energy). The value of $E_{\mathrm{a}}$ obtained from the slope was $(43 \pm 5) \mathrm{kJ} \mathrm{mol}^{-1}$ which shows good agreement (within error) with that reported previously $\left(44 \mathrm{~kJ} \mathrm{~mol}^{-1}\right),{ }^{12}$ establishing the principle of disc MSM LKE activation energy measurement.

In fact, it should also be possible to extract values for $D$ and $c$ from analysing the shape of the chronoamperometric transients for disc MSMs. If one avoids fitting the currents obtained in the first few tens of milliseconds, where double layer charging dominates the current, Xiong et al. ${ }^{34}$ have shown satisfactory fits to the equation developed by Shoup and Szabo: ${ }^{35}$

$$
i(t)=4 n F D c r\left(A^{\prime}+B^{\prime} \frac{r}{\sqrt{D t}}+C^{\prime} \exp \left(-D^{\prime} \frac{r}{\sqrt{D t}}\right)\right)
$$

where $A^{\prime}=\frac{\pi}{4}, B^{\prime}=\frac{\sqrt{\pi}}{4}, C^{\prime}=1-\frac{\pi}{4}$ and $D^{\prime}=\frac{\frac{\sqrt{\pi}}{4}-2 \pi^{-3 / 2}}{1-\frac{\pi}{4}}$ for redox couples in non-aqueous solvents and ionic liquids, particularly for data recorded over the first few seconds of the transient.

Fig. 4A shows a comparable chronoamperometric transient recorded in aqueous media, when using an $r=25 \mu \mathrm{m}$ Pt MSM to study $5 \mathrm{mM}$ ferricyanide in $100 \mathrm{mM}$ aqueous $\mathrm{KCl}$. Consistent with Xiong et al., it is clear that a good fit of eqn (3) to the data is observed if one limits the fit to the data recorded between $0.05 \mathrm{~s}$ and $1 \mathrm{~s}$. The adjusted $R^{2}$ value was found to be 0.9988 and physically realistic values for ferrocyanide of $c=5.6 \pm 0.1 \mathrm{mM}$ and $D=(4.2 \pm 0.1) \times 10^{-6} \mathrm{~cm}^{2} \mathrm{~s}^{-1}$ were obtained. Also included in the plot is a fit to eqn (4), the established equation for the transient current at a hemispherical electrode. ${ }^{34}$

$$
i=\frac{n F A D^{1 / 2} c}{\pi^{-1 / 2} t^{-1 / 2}}+4 n F D c r
$$

where $A=\pi r^{2}$ is the area of the electrode. This simpler equation, which models the expected Cottrell behaviour at short times with the first term, and the steadystate mass transport limited current from eqn (2) at long times with the second term, would be expected to suffer from inaccuracy in the transition region due to
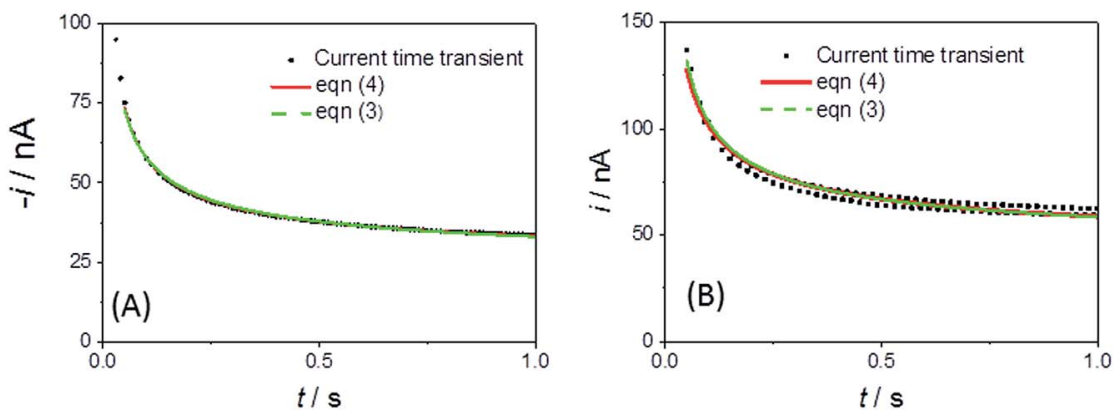

Fig. 4 Current-time transient and fits to eqn (3) and (4) for an $r=25 \mu \mathrm{m} \mathrm{MSM} \mathrm{Pt} \mathrm{disc} \mathrm{in} \mathrm{(A)}$ $100 \mathrm{mM}$ aqueous $\mathrm{KCl}$ with $5 \mathrm{mM}$ ferricyanide ( $E$ jumped from +0.34 to $0.14 \mathrm{~V}$ at $t=0$ ) and (B) LKE with $19 \mathrm{mM}$ added $\mathrm{EuCl}_{3}$ ( $E$ jumped from +0.5 to $+0.2 \mathrm{~V}$ at $t=0$ ). 
the switch from planar diffusional symmetry at short times to hemispherical diffusional symmetry at longer times. Interestingly it gives almost as good a fit as eqn (3), with adj. $R^{2}=0.9975, c=5.2 \pm 0.1 \mathrm{mM}$ and $D=(4.3 \pm 0.9) \times 10^{-6} \mathrm{~cm}^{2}$ $\mathrm{s}^{-1}$.

Fig. 4B shows the typical corresponding fits to the current-time transient recorded with $\mathrm{EuCl}_{3}$ in LKE. In this case the fit to the Shoup-Szabo eqn (3) was again reasonable, with $D=(4.4 \pm 0.3) \times 10^{-6} \mathrm{~cm}^{2} \mathrm{~s}^{-1}$, which is consistent with the global value of $D, c=9.5 \pm 0.4 \mathrm{mM}$ and adj. $R^{2}=0.9694$. The fit to eqn (4) again produced comparable parameters with $c=8.7 \pm 0.5 \mathrm{mM}$ and $D=(4.3 \pm 0.3)$ $\times 10^{-6} \mathrm{~cm}^{2} \mathrm{~s}^{-1}$ and was only marginally worse with adj. $R^{2}=0.9655$.

3.2.2 Electrochemical impedance spectroscopy (EIS). We have previously shown that accurate quantitative data can be obtained from electrochemical impedance spectroscopy (EIS) for square MSMs using solution redox couples under ambient aqueous conditions. ${ }^{29}$

Fig. 5A shows the typical EIS response for a $r=25 \mu \mathrm{m}$ Pt disc MSM to an aqueous $5 \mathrm{mM}$ potassium ferricyanide solution at $293 \mathrm{~K}$. It is reassuring that the response fits well (with $\chi^{2}=0.002$ ) to the established modified Randles' equivalent circuit for the disc microelectrode ${ }^{36-38}$ (Fig. 5C), confirming its applicability to ambient aqueous measurements with disc MSMs as with other disc microelectrodes.

Fig. 5B shows the corresponding response of an MSM to the Eu(III/II) couple at its formal potential in LKE solution at $703 \mathrm{~K}$. For these and all other EIS LKE disc MSM data, the best fit was achieved by replacing the Warburg element with
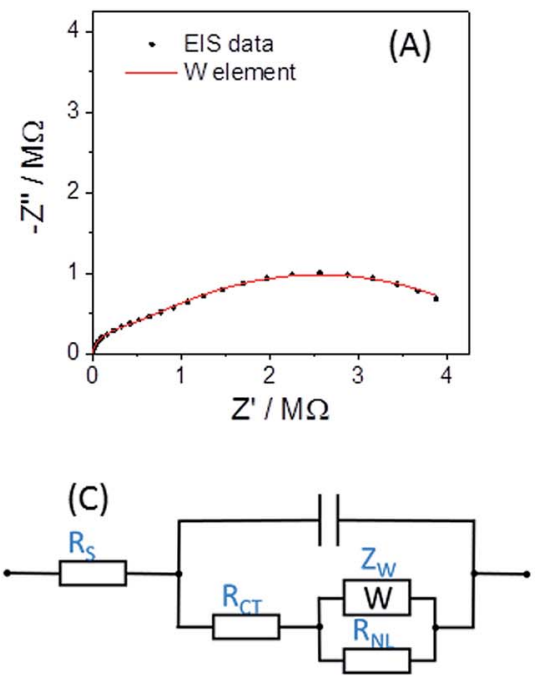
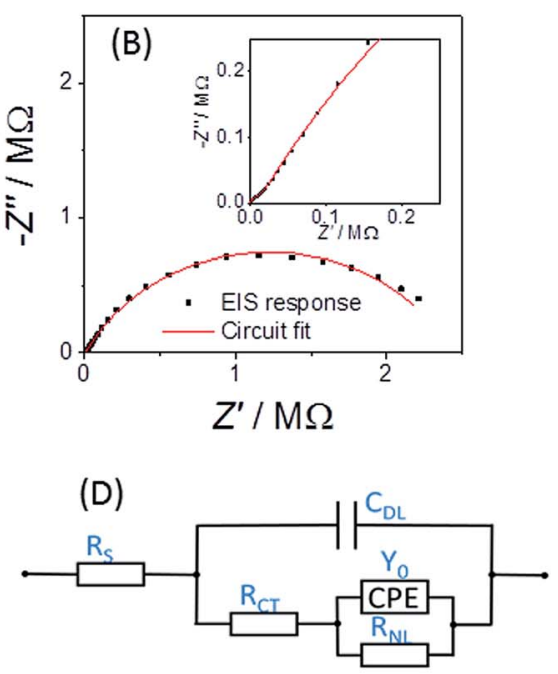

Fig. 5 (A) EIS response at $E_{\mathrm{DC}}=+0.240 \mathrm{~V}$ (points) and equivalent circuit fit (line) for an $r=$ $25 \mu \mathrm{m}$ Pt disc electrode in $100 \mathrm{mM}$ aqueous $\mathrm{KCl}$ containing $5 \mathrm{mM}$ potassium ferrocyanide. (B) EIS response at $E_{\mathrm{DC}}=+0.345 \mathrm{~V}$ (points) for an $r=25 \mu \mathrm{m}$ Pt disc and equivalent circuit fits (lines) in $19 \mathrm{mM}$ added $\mathrm{EuCl}_{3}$ in LKE at $703 \mathrm{~K}$. The inset shows an expansion of the higher frequency data, showing the greater than $45^{\circ}$ slope consistent with a CPE. (C) The established modified Randles' circuit employed for fitting aqueous disc microelectrode EIS data. (D) The proposed modified Randles' circuit employed for fitting disc MSM EIS data in LKE MS. 
a constant phase element (CPE), which is required to fit the gradient of greater than $45^{\circ}$ observed at higher frequencies in the Nyquist plot (see inset to Fig. $5 \mathrm{~B}$ ). The fitted values for these data were: $C_{\mathrm{DL}}=1.66 \pm 0.02 \mathrm{nF} ; R_{\mathrm{CT}}=1590 \pm 50 \mathrm{k} \Omega ; Q_{0}$ $=(1.12 \pm 0.03) \times 10^{-7} \mathrm{~S} \mathrm{~s}^{n} ; n=0.695 \pm 0.005 ; R_{\mathrm{NL}}=2.42 \pm 0.04 \mathrm{M} \Omega$, with $\chi^{2}=$ 0.03. It is interesting that the equivalent data recorded in the same LKE solution using a bare tungsten macrowire electrode also requires the use of a constant phase element in place of the Warburg element in the Randles' circuit, and that the fitted value of $n=0.69 \pm 0.03$ is the same within experimental error as that observed for the MSM in LKE. This suggests a fundamental difference in the linear diffusional properties of ambient aqueous and LKE MS solution at these frequencies. Cuervo-Reyes et al. ${ }^{39}$ have recently interpreted a diffusional CPE as being due to subdiffusive transport and local trapping of charge carriers. It is tempting to infer that some of the strong electrostatic coupling and local mobile ion trapping postulated in this work in batteries and mobile glasses may also be present to some extent in LKE MS, and that this may also be the origin of the observed CPE.

Turning to the other measured EIS parameters, we have previously shown for square ambient aqueous microelectrodes that at the formal potential of a oneelectron redox couple the minimum values of the two resistances $R_{\mathrm{CT}}$ and $R_{\mathrm{NL}}$ should be given by: ${ }^{29}$

$$
R_{\mathrm{NL}}^{\min }=\frac{4 R T}{n F i_{\mathrm{L}}}
$$

and

$$
R_{\mathrm{CT}}^{\min }=\frac{2 R T}{n^{2} F^{2} c A k^{0}}
$$

with the variation of each with $E_{\mathrm{DC}}$ being given by:

$$
R_{\mathrm{NL}}=\frac{R_{\mathrm{NL}}^{\min }}{4}\left[2+\exp \left(\frac{-F\left(E_{\mathrm{DC}}-E^{0}\right)}{R T}\right)+\exp \left(\frac{F\left(E_{\mathrm{DC}}-E^{0}\right)}{R T}\right)\right]
$$

and
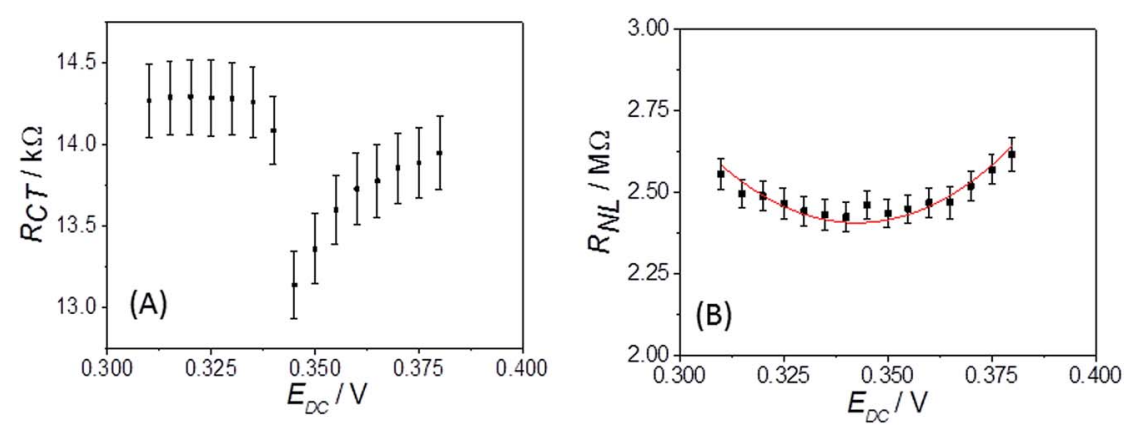

Fig. 6 Extracted values of (A) $R_{\mathrm{CT}}$ and (B) $R_{\mathrm{NL}}$ with errors from iterative fits of the EIS response of the $r=25 \mu \mathrm{m}$ Pt disc electrode in $19 \mathrm{mM} \mathrm{EuCl}_{3} \mathrm{LKE}$ to the modified Randles' circuit (Fig. 5D). (B) also shows the fit (red line) of these values to eqn (7). 


$$
R_{\mathrm{CT}}=\frac{R_{\mathrm{CT}}^{\min }}{2}\left[\exp \left(\frac{-\alpha_{\mathrm{ox}} F\left(E_{\mathrm{DC}}-E^{0}\right)}{R T}\right)+\exp \left(\frac{\left(1-\alpha_{\mathrm{ox}}\right) F\left(E_{\mathrm{DC}}-E^{0}\right)}{R T}\right)\right]
$$

where $E^{0}$ is the formal potential and $\alpha_{\mathrm{ox}}$ is the transfer coefficient for the oxidation reaction.

It is clear from Fig. 6B that there is good agreement between the disc MSM data and eqn (7), and the fitted value of $R_{\mathrm{NL}}^{\mathrm{min}}=2.42 \pm 0.02 \mathrm{M} \Omega$ also agrees well with the value of $2.41 \pm 0.03 \mathrm{M} \Omega$ calculated from the data in Fig. 3A and B using eqn (5). This indicates that the established EIS MSM theory (eqn (5)-(8)) can be used to extract quantitative information from disc MSM measurements in LKE MS. Using the observed value of $R_{\mathrm{CT}}^{\min }=13.1 \pm 0.2 \mathrm{k} \Omega$ a value of $k^{0}=0.26 \mathrm{~cm} \mathrm{~s}^{-1}$ can be extracted from eqn (6). ${ }^{29}$ This value is two orders of magnitude faster than that previously reported in the literature for this system $\left(3.31 \times 10^{-3} \mathrm{~cm} \mathrm{~s}^{-1}\right.$ on a glassy carbon macroelectrode). ${ }^{40}$ This is consistent with previous ambient aqueous microelectrode measurements, which have demonstrated that reduction of the dimensions of the electrode to the micron scale allows for markedly more efficient mass transport and the measurement of more realistic charge transfer rates. ${ }^{41}$ Given this, it is interesting that the $R_{\mathrm{CT}}$ data (Fig. $6 \mathrm{~A}$ ) shows the predicted minimum at $E_{\mathrm{DC}}=E^{0}$, but that these data do not fit well to eqn (8). Although the errors on these data are relatively large, this may suggest some difference between charge transfer behaviour in LKE MS and in ambient aqueous solution.

\subsection{Extending the nature of disc MSM measurements - electroanalysis to characterise the formation of metal alloys}

Bismuth is a metal of importance within nuclear pyrochemical processing. It melts at $271.4{ }^{\circ} \mathrm{C}$ and liquid bismuth electrodes are well known for their propensity to form alloys when electroreducing actinide and lanthanide species dissolved in LKE. ${ }^{42}$ As a result of these properties, bismuth is one of the candidates to be used as a reactive liquid metal cathode for the selective extraction of fuel species as metal alloys during the electro-refining stage of nuclear fuel reprocessing..$^{43}$ In this study, our aim was to observe whether electrochemical Bi alloying reactions could be studied using the disc MSM.

Fig. 7A shows typical CVs of the behaviour of the bismuth(III/0) couple on Pt and W disc MSM electrodes. When cycling the W MSM electrodes, a simple plating wave (for Bi(III) reduction and deposition) and essentially single stripping peak (for $\mathrm{Bi}(0)$ oxidation and dissolution) were observed, centred near $E=-0.06$ $\mathrm{V}$. This is consistent with the phase diagram, which indicates that $\mathrm{W}$ and Bi do not form alloys. Using eqn (1) and the observed mass transport limited reduction current, the diffusion coefficient for $\mathrm{Bi}^{3+}$ was determined to be $(6.7 \pm 0.02) \times 10^{-6}$ $\mathrm{cm}^{2} \mathrm{~s}^{-1}$ at $698 \mathrm{~K}$. In contrast, the Pt MSMs showed $\mathrm{Bi}(\mathrm{III}) /(0)$ plating and associated stripping electrochemistry, centred slightly above $0 \mathrm{~V}$, with three identifiable, overlapping stripping peaks. It is satisfactory that there is very close agreement for the values of $i_{\mathrm{L}}$ found for the Pt and W MSM electrodes (a 2.1\% variation in $i_{\mathrm{L}}$, which is within the error for the devices), which demonstrates that these differences are not due to changes in $n$ or $A$, but rather to the effects of $\mathrm{Pt} / \mathrm{Bi}$ alloying.

The Pt-Bi phase diagram ${ }^{44}$ indicates two thermodynamically stable solid alloy phases under these conditions $\left(\mathrm{PtBi}_{2}\right.$ and $\left.\mathrm{PtBi}\right)$, with a third state of intermediate composition $\left(\mathrm{Pt}_{2} \mathrm{Bi}_{3}\right)$ which is thermodynamically stable above $843 \mathrm{~K}$. We 

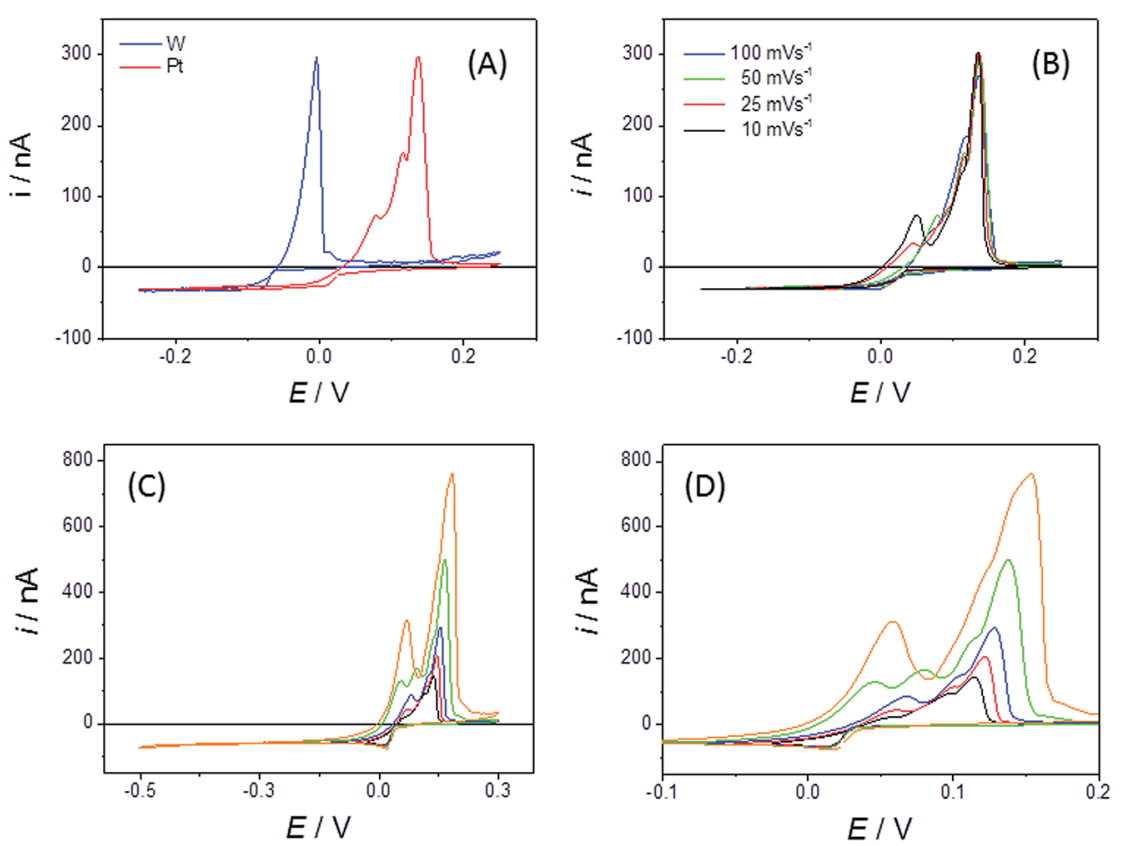

Fig. 7 (A) CVs recorded using a sweep rate of $50 \mathrm{mV} \mathrm{s}^{-1}$ with an $r=25 \mu \mathrm{m}$ Pt and an $r=25$ $\mu \mathrm{m} \mathrm{W} \mathrm{MSM}$ in LKE $+1.6 \mathrm{mM} \mathrm{BiCl}_{3}$ at $668 \mathrm{~K}$. (B) CVs recorded at the given sweep rates using an $r=15 \mu \mathrm{m}$ diameter Pt MSM in LKE $+5 \mathrm{mM} \mathrm{BiCl}_{3}$ at $690 \mathrm{~K}$. (C) CV and (D) expanded section of the $C V$ at $50 \mathrm{mV} \mathrm{s}^{-1}$ with a progressively increasing negative potential limit recorded using a Pt MSM $(r=25 \mu \mathrm{m})$ in LKE $+1.6 \mathrm{mM} \mathrm{BiCl}_{3}$.

tentatively propose these three stripping peaks originate from stable $\mathrm{PtBi}_{2}$, metastable $\mathrm{Pt}_{2} \mathrm{Bi}_{3}$ and stable $\mathrm{PtBi}$ phases respectively; further quantitative experiments are underway to establish whether this is the case. Fig. 7B also shows that when a Pt disc MSM electrode is swept over a range of scan rates a fourth stripping peak is increasingly observed at the most cathodic potential at the slowest scan rates. This we attribute to stripping of the stable liquid metal alloy (predominantly Bi) phase expected from the phase diagram at high Bi concentrations. Additionally, Fig. 7C and D show the effect of progressively increasing the negative potential limit of the CV scan (which also increases the deposition time). In this case all peaks increase in size, consistent with increased Bi deposition and alloy formation, and unlike in Fig. 7B, the peaks shift progressively to more positive potentials, indicating the contribution of stripping kinetics at these increased coverages. That significant $\mathrm{Bi}$ is deposited was confirmed by visual inspection following prolonged plating (Fig. 8C and D). The maximum thickness of liquid Bi plated across the $r=25 \mu \mathrm{m}$ Pt disc surface, at the slowest sweep rate, was calculated to be $\sim 9 \mathrm{~nm}$. It is interesting that the reduction to bismuth metal takes place at a higher potential on the MSM than on a Pt wire macroelectrode (Fig. 8A), and distinct stripping peaks were not observed when using this macrowire, even though the calculated amount of Bi deposited per unit area was similar on each electrode. This indicates greater richness in Bi alloy characterisation information when using MSMs. 

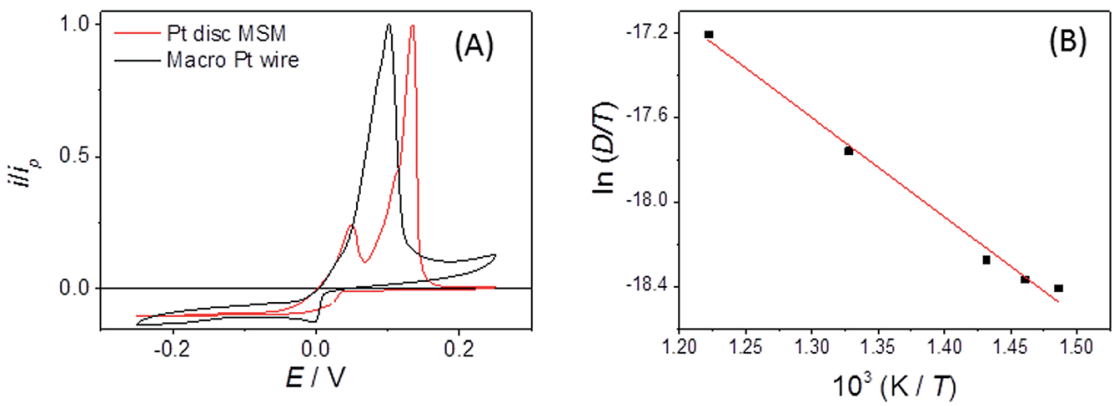

(C)

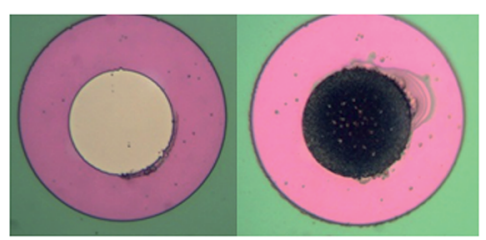

(D)

Fig. 8 (A) CVs normalised to the peak stripping current $\left(i_{p}\right)$ recorded using a Pt wire macroelectrode and an $r=25 \mu \mathrm{m} \mathrm{Pt}$ disc at a sweep rate of $10 \mathrm{mV} \mathrm{s}^{-1}$. (B) Arrhenius plot used to obtain the activation energy for the diffusion of $\mathrm{Bi}^{3+}$ in LKE. (C) Image of an $r=25$ $\mu \mathrm{m}$ Pt disc before immersion in LKE $+1.5 \mathrm{mM} \mathrm{BiCl}_{3}$. (D) Image of the same $r=25 \mu \mathrm{m} \mathrm{Pt}$ disc electrode following application of a potential of $-0.2 \mathrm{~V}$ for $120 \mathrm{~s}$ in $\mathrm{LKE}+1.5 \mathrm{mM} \mathrm{BiCl}_{3}$ to deposit $\mathrm{Bi}$.

As with $\mathrm{Eu}(\mathrm{III}) /(\mathrm{II})$ above, the values of $i_{\mathrm{L}}$ observed for Bi(III) plating on the disc MSMs were used in eqn (1) to obtain Bi(III) diffusion coefficients, from which an Arrhenius plot was constructed (Fig. 8B). The resulting value of $E_{\mathrm{a}}=39 \pm 4 \mathrm{~kJ}$ $\mathrm{mol}^{-1}$ was in close agreement with that found for Eu.

Bismuth binary and ternary alloys that do not involve Pt (of potential importance when carrying out coextraction using Bi electrodes) can also be studied by using W disc MSMs. Fig. 9 shows typical CVs for a mixed Bi(III), Zn(II) system in LKE, which shows $\mathrm{Bi}(\mathrm{III}) /(0)$ plating/stripping (near $E=0 \mathrm{~V}$ ), $\mathrm{Zn}(\mathrm{II}) / \mathrm{Zn}(0)$ plating stripping (near $E=-0.9 \mathrm{~V}$ ) and $\operatorname{Li}(\mathrm{I}) /(0)$ plating/stripping (at and below $E=-1.8$
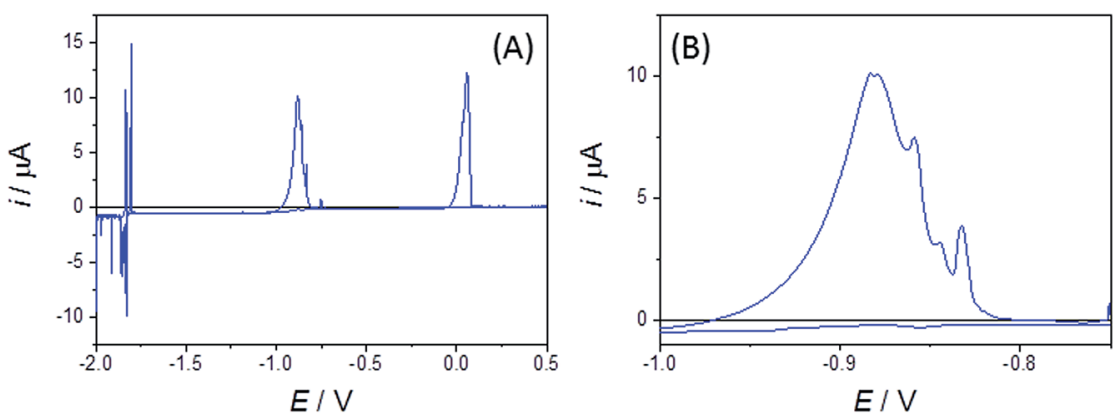

Fig. 9 (A) Bismuth, zinc and lithium codeposition studies using CV (at a sweep rate of 50 $\mathrm{mV} \mathrm{s}^{-1}$ ) in LKE $+5 \mathrm{mM} \mathrm{BiCl}_{3}+10 \mathrm{mM} \mathrm{ZnCl}_{2}$ recorded using an $r=25 \mu \mathrm{m} \mathrm{W} \mathrm{MSM}$. (B) Expanded section of the $\mathrm{CV}$ showing distinct peaks in the $\mathrm{Zn}$ stripping region attributed to stripping from alloy phases. 
V). The distinct peaks observed in $\mathrm{Li}(\mathrm{I}) /(0)$ plating/stripping and $\mathrm{Zn}(0)$ stripping features are again attributed to alloy formation, and further indicate the richness of alloying information provided by these disc MSMs.

\section{Conclusions}

This work has demonstrated the feasibility of fabrication of Pt and W disc MSMs and their stability in, and their potential for, quantitative characterisation of, the LKE MS system. Much of the information that can be obtained is comparable to that obtained from ambient aqueous studies using these or other disc microelectrodes, with values of $E^{0}, D, c$ and $E_{\mathrm{a}}$ being readily obtainable from established electrochemical methods using analysis established for aqueous systems. As with aqueous measurements, it appears that the enhanced mass transport rates at disc MSMs lead to the measurement of markedly larger and more realistic electrochemical charge transfer rate constants, $k^{0}$, than those observed at macroelectrodes.

In addition to these established similarities to aqueous disc MSM responses, which demonstrate the potential of disc MSMs in enhanced MS sensing, analysis and process development, there are also some interesting differences. Whilst a minimum is observed at $E^{0}$ for $R_{\mathrm{CT}}$ in EIS, the shape of the variation with $E_{\mathrm{DC}}$ is apparently not as predicted by ambient aqueous theory. There are hints from variation in the EIS equivalent circuit that there may be a contribution from subdiffusive transport in the LKE MS, as seen previously in glass melts and Li-ion battery materials. There is also the marked enhancement of characterisation information for metal alloy formation from LKE MS compared to macroelectrodes. These measurements provide real motivation for additional study to gain further physicochemical insight into the properties of LKE and other MS systems.

\section{Acknowledgements}

The authors acknowledge financial support from, and development of this work through, the UK EPSRC REFINE project (EP/J000779/1) and the EC SACSESS (Contract No. FP7-Fission-2012-323282) and SMART Microsystems (FS/01/02/10 IeMRC Flagship) programmes. We also acknowledge EPSRC and National Nuclear Laboratory (NNL) financial support for studentships. Data used within this publication can be accessed at (http://dx.doi.org/10.7488/ds/1338).

\section{References}

1 G. Z. Chen, D. J. Fray and T. W. Farthing, Nature, 2000, 407, 361.

2 K. Sridharan, Thermal Properties of LiCl-KCl Molten Salt for Nuclear Waste Separation, Project No. 09-780, Nuclear Energy University Programs, US Department of Energy, 2012.

3 H. P. Nawada and K. Fukuda, J. Phys. Chem. Solids, 2005, 66, 647.

4 D. D. Sood and S. K. Patil, J. Radioanal. Nucl. Chem., 1996, 203, 547.

5 T. Inoue and L. Koch, Nucl. Eng. Technol., 2008, 40, 183-190.

6 K. Xu, Chem. Rev., 2004, 104, 4303. 
7 D. Hamon, M. Vrinat, M. Breysse, B. Durand, M. Jebrouni, M. Roubin, P. Magnoux and T. des Courieres, Catal. Today, 1991, 10, 613.

8 G. W. Parshall, J. Am. Chem. Soc., 1972, 94, 8716.

9 S. Y. Oh, J. Y. Kim, S. E. Bae, Y. H. Cho, J. W. Yeon and K. Song, J. Lumin., 2013, 134, 706.

10 C. A. Schroll, S. Chatterjee, T. G. Levitskaia, W. R. Heineman and S. A. Bryan, Anal. Chem., 2013, 85, 9924.

11 A. Uehara, T. Nagai, T. Fujii, O. Shirai and H. Yamana, J. Nucl. Mater., 2013, 437, 166.

12 C. Caravaca, G. Cordoba and M. J. Tomas, Electrochemical study of europium trichloride in molten eutectic LiCl-KCl2005, https://oecd-nea.org/pt/docs/iem/ lasvegas04/09_Poster_Session_I/PS1_01.pdf.

13 Y. Castrillejo, P. Herníndez, J. A. Rodriguez, M. Vega and E. Barrado, Electrochim. Acta, 2012, 71, 166.

14 G. Cordoba and C. Caravaca, J. Electroanal. Chem., 2004, 572, 145.

15 S. Ghosh, S. Vandarkuzhali, P. Venkatesh, G. Seenivasan, T. Subramanian, B. Prabhakara Reddy and K. Nagarajan, J. Electroanal. Chem., 2009, 627, 15.

16 T. J. Kim, Y. K. Jeong, J. G. Kang, Y. Jung, D. H. Ahn and H. S. Lee, J. Radioanal. Nucl. Chem., 2010, 286, 283.

17 K. Kushkhov, M. K. Vindizheva, R. A. Karashaeva and M. R. Tlenkopachev, Russ. J. Electrochem., 2010, 46, 691.

18 H. A. Laitinen, C. H. Liu and W. S. Ferguson, Anal. Chem., 1958, 30, 1266.

19 P. Masset, D. Bottomley, R. Konings, R. Malmbeck, A. Rodrigues, J. Serp and J. P. Glatz, J. Electrochem. Soc., 2005, 152, A1109.

20 B. Prabhakara Reddy, S. Vandarkuzhali, T. Subramanian and P. Venkatesh, Electrochim. Acta, 2004, 49, 2471.

21 D. K. Corrigan, E. O. Blair, J. G. Terry, A. J. Walton and A. R. Mount, Anal. Chem., 2014, 86, 11342.

22 R. J. Forster, Chem. Soc. Rev., 1994, 23, 289.

23 J. Heinze, Angew. Chem., Int. Ed., 1993, 32, 1268.

24 M. Sosna, G. Denuault, R. W. Pascal, R. D. Prien and M. Mowlem, Sens. Actuators, B, 2007, 123, 344.

25 S. Legeai, K. k. Soropogui, M. Cretinon, O. Vittori, A. Heeren De Oliveira, F. d. r. Barbier and M. F. Grenier-Loustalot, Anal. Bioanal. Chem., 2005, 383, 839.

26 R. T. Carlin and R. A. Osteryoung, J. Electrochem. Soc., 1989, 136, 1249.

27 E. O. Blair, D. K. Corrigan, J. G. Terry, A. R. Mount and A. J. Walton, J. Microelectromech. Syst., 2015, 24, 1346.

28 A. Relf, D. Corrigan, C. L. Brady, J. G. Terry, A. J. Walton and A. R. Mount, ECS Trans., 2013, 50, 105.

29 H. L. Woodvine, J. G. Terry, A. J. Walton and A. R. Mount, Analyst, 2010, 135, 1058.

30 C. G. Zoski and M. V. Mirkin, Anal. Chem., 2002, 74, 1986.

31 G. J. Janz and N. P. Bansal, J. Phys. Chem. Ref. Data, 1982, 11, 505.

32 S. A. Kuznetsov and M. Gaune-Escard, J. Electroanal. Chem., 2006, 595, 11.

33 A. Einstein, Ann. Phys., 1905, 17, 549.

34 L. Xiong, L. Aldous, M. C. Henstridge and R. G. Compton, Anal. Methods, 2012, 4, 371.

35 D. Shoup and A. Szabo, J. Electroanal. Chem., 1982, 140, 237. 
36 P. Los, G. Zabinska, A. Kisza, L. Christie, A. Mount and P. G. Bruce, Phys. Chem. Chem. Phys., 2002, 2, 5449.

37 P. Los and P. G. Bruce, Pol. J. Chem., 1997, 71, 1151.

38 M. Fleischmann, S. Pons and J. Daschbach, J. Electroanal. Chem., 1991, 317, 1.

39 E. Cuervo-Reyes, C. P. Scheller, M. Held and U. Sennhauser, J. Electrochem. Soc., 2015, 162, A1585.

40 M. R. Bermejo, F. de la Rosa, E. Barrado and Y. Castrillejo, J. Electroanal. Chem., 2007, 603, 81.

41 I. Schmueser, A. J. Walton, J. G. Terry, H. L. Woodvine, N. J. Freeman and A. R. Mount, Faraday Discuss., 2013, 164, 295.

42 M. Kurata, Y. Sakamura and T. Matsui, J. Alloys Compd., 1996, 234, 83.

43 W. Dong, X. Yang, J. Zhang and J. Sheng, J. Mater. Sci., 2008, 43, 3240.

44 H. Okamoto, J. Phase Equilib., 1991, 12, 207. 